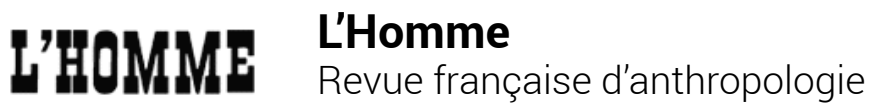

$233 \mid 2020$

Varia

\section{Charlotte Marchina, Nomad's Land. Éleveurs, animaux et paysage chez les peuples mongols}

Jean-Pierre Digard

\section{(2) OpenEdition}

Édition électronique

URL : https://journals.openedition.org//homme/36931

DOI : 10.4000//homme.36931

ISSN : 1953-8103

Éditeur

Éditions de l'EHESS

\section{Édition imprimée}

Date de publication : 27 février 2020

Pagination : 171-173

ISBN : 9782713228360

ISSN : 0439-4216

\section{Référence électronique}

Jean-Pierre Digard, «Charlotte Marchina, Nomad's Land. Éleveurs, animaux et paysage chez les peuples mongols ", L'Homme [En ligne], 233 | 2020, mis en ligne le 27 février 2020, consulté le 04 janvier 2023

URL : http://journals.openedition.org//homme/36931; DOI : https://doi.org/10.4000//homme.36931 



\section{ASIE \& OCÉANIE}

\section{Charlotte Marchina}

Nomad's Land. Éleveurs, animaux et paysage chez les peuples mongols

Le Kremlin-Bicêtre, Zones sensibles, 2019, 224 p., bibl., index, ill., fig., cartes

(«Pactum serva »).

$F$

RUIT DE vingt mois d'enquête cumulés depuis 2008 en Mongolie et en Russie, ce livre décrit et compare les modes de vie de deux ethnies mongolophones: les Halh en République populaire de Mongolie et les Bouriates en Sibérie méridionale (Fédération de Russie). Les uns et les autres se disent, non pas «nomades», terme auquel ils semblent attribuer une connotation péjorative, mais "éleveurs qui nomadisent». Leur activité de base est l'élevage extensif et conjoint des "cinq museaux» (chameaux, chevaux, bovins, moutons et chèvres), sans oublier les chiens, dans un environnement de steppe (chap. I: «Nomads's land, no man's land?»). Dans ce contexte, leur «mobilité est un moyen de maintenir l'équilibre entre les ressources et la pression qu'y exercent les éleveurs et leurs animaux» (p. 29). Mais, loin d'être uniforme, cette mobilité présente de multiples modalités (carte 6, pp. 42-43) et états intermédiaires entre nomadisme, migration, transhumance et sédentarisation, de même qu'entre appropriation collective et appropriation privée des terres, le bétail relevant, lui, presque toujours de la propriété privée.

La pratique du nomadisme, ici comme ailleurs, n'implique pas que l'on puisse circuler n'importe où. Bien au contraire, le territoire de nomadisation (nutag ou nuytag) est celui où l'on est né et dont il n'est pas bon de trop s'éloigner, au risque de voir les animaux fuguer (chap. II : "Tenir et appartenir à son pays»). Faire marcher le bétail, c'est activer des "forces centripètes» (p. 81). Les sentiers (jim) creusés par les pas des animaux sont la matérialisation d'une routine, mais aussi un encouragement à cette routine. D’autres moyens, matériels et immatériels, sont mis en œuvre pour resserrer les liens avec les nutag: érection de cairns de pierres (oboo), dépôt de crânes de chevaux en des endroits élevés, cultes rendus aux "esprits maîtres des lieux" (gazryn ezen), rituels de consécration d'animaux (chevaux surtout) effectués par des chamanes - tout cela pour signifier: "We are here!", selon l'heureuse expression de l'anthropologue britannique Caroline Humphrey (p. 111).

Comment les différentes espèces animales cohabitent-elles dans les espaces du nutag? (chap. III : «Espaces d'espèces»). Dans ce type d'élevage, les pâturages ne sont pas clôturés, à l'exception de ce que Carole Ferret nomme les «exclos» (p. 126), conçus pour empêcher d'autres animaux d'approcher. Qualité prisée entre toutes, l'autonomie des herbivores sur les pâturages varie selon les espèces: les chameaux et, surtout, les chevaux sont les plus autonomes et donc considérés comme les plus «intelligents» (uhaantai), à la différence des petits ruminants, plus vulnérables aux attaques des prédateurs - loups, "vautours" (vraiment?, p. 120) - et qui 
doivent, par conséquent, être "gardiennés" (pasti) en permanence, de manières et à des traites, ainsi que les jeunes non sevrés sont gardés à proximité des campements.

Les troupeaux communs à plusieurs propriétaires sont l'exception. Les uniques lieux de rencontre et de partage entre les éleveurs sont les points d'eau; comme pour les pâturages, la présence d'espèces différentes permet leur utilisation optimale, surtout quand il gèle: on fait alors passer les chevaux en premier, afin qu'ils cassent la glace pour le petit bétail. Les espaces des campements sont réservés aux habitations humaines, puis, autour d'elles, aux animaux de monte, aux femelles traites et aux animaux jeunes ou fragiles. Les campements d'été se distinguent de ceux d'hiver par la prépondérance des éléments amovibles (yourtes, palissades, cordes d'attache, etc.).

Le chapitre IV ("Animaux et territoires») contient un intéressant "interlude canin" (pp. 175-181), qui met l'accent sur le cantonnement des chiens - des molosses d'un type (banhar) proche du dogue tibétain, en réalité issus de multiples croisements dans une fonction de défense des campements et du cheptel. Sur ce sujet comme sur celui du chapitre précédent, on ne peut manquer d'être frappé par les similitudes qui existent entre les Mongols décrits ici et d'autres sociétés pastorales, en particulier les nomades montagnards d'Afrique du Nord et du Moyen-Orient, similitudes qu'il aurait sans doute été intéressant d'interroger ${ }^{1}$.

La question, examinée ensuite, des races (ü̈̈lder) et des types (omog) du bétail mongol reste à peu près entière faute d'étude détaillée des stratégies de reproduction animale mises en œuvre par les éleveurs, à l'exception de quelques exemples de sélection à l'intérieur de "races de sang" (plemya) pour les chevaux ou, au contraire, de croisement de races locales (mestnye) avec des races européennes, comme la holstein, pour améliorer les qualités laitières des vaches mongoles. Le métissage entre races est aussi étudié pour les chevaux (en mongol, le même terme, erliiz, désigne

les animaux métis et les personnes issues d'union bi-ethniques). Est également pratiqué le croisement vache $\mathrm{x}$ yak : les hybrides (hainag) donnent autant de lait que la vache, mais sont plus robustes et vivent plus longtemps (des photographies descriptives de ces différents types, races et espèces auraient été les bienvenues). Pour le reste, les préférences locales vont aux animaux qui montrent le plus de rusticité, d'autonomie, d'aptitude à subvenir seuls à leurs besoins, à élever leurs petits, etc., sur les pâturages locaux, en réduisant d'autant les contraintes de leur affouragement et de leur gardiennage.

En conclusion, Charlotte Marchina dégage les principaux traits qui distinguent les deux groupes étudiés. Chez les Halh de Mongolie, ce sont: élevage à orientation domestique, propriété publique ou collective des pâturages permettant une grande flexibilité des itinéraires de nomadisation, discours officiels mettant en avant " une nation envisagée comme une relation triadique symbiotique entre humains, environnement et animaux, notamment à travers l'élargissement du nutag, originellement défini par le territoire de nomadisation et de pâturage» (p. 203). Chez les Bouriates de Sibérie, ce sont: élevage à orientation marchande, propriété privée des terres, transformation des éleveurs en éleveurs-agriculteurs dans le cadre d'exploitations prenant des allures de ranch, nostalgie de l'ancien genre de vie. Les deux derniers paragraphes de la conclusion (pp. 205-206) consistent en une profession de foi descolienne sur le thème de la remise en cause de la distinction nature/culture - profession de foi dont on ne discerne pas bien le rapport

1. Cf., pour se limiter au domaine cynotechnique: Xavier de Planhol, «Le chien de berger: développement et signification géographique d'une technique pastorale", Bulletin de l'Association de géographes français, 1969, 370: 355-368 (https://www.persee.fr/ doc/bagf_0004-5322_1969_num_46_370_5897); Jean-Pierre Digard, "Chiens de campement et chiens de troupeau chez les nomades Baxtyâri d'Iran ", Studia iranica, 1980, 9/1:131-139, et "Les fonctions du chien chez les pasteurs nomades des montagnes de l'Asie du Sud-Ouest», Ethnozootechnie, 2006, 78: 125-129. 
avec ce qui précède et qui arrive donc un peu comme un cheveu sur la soupe.

Mais le plus important n'est pas là. L'originalité principale du travail de Charlotte Marchina tient à la technique d'enregistrement des déplacements des troupeaux qu'elle a mise en œuvre en équipant des animaux de balises GPS, enregistrement à partir duquel le Studio Joost Grootens d'Amsterdam a réalisé une série de cartes originales, précises et détaillées. Or, l'auteure ne tire pas tout le bénéfice que l'on aurait pu attendre de cet extraordinaire matériel cartographique, qui se trouve, de ce fait, réduit à un rôle presque exclusivement illustratif. Pour aller plus loin, il lui aurait fallu connaitre le travail d'inventaire des nomadismes qui fut effectué au milieu du siècle dernier par les géographes français du Sahara ${ }^{2}$. Elle aurait aussi pu inscrire sa cartographie dans le prolongement d'autres travaux, moins anciens, comme la typologie des formes de nomadisme tentée par Douglas Johnson ${ }^{3}$. De même, le vaste tableau synthétique des déplacements pastoraux en Asie centrale élaboré plus récemment par Carole Ferret ${ }^{4}$ figure bien dans la bibliographie, mais sans être véritablement mis à contribution.
En somme, et malgré ces quelques déficits comparatistes, l'ouvrage de Charlotte Marchina, documenté de première main et que complètent une bibliographie de quelque 150 titres et un index de 90 entrées, restera sans doute longtemps, sur le nomadisme mongol, ses variantes et leurs transformations contemporaines, une source incontournable.

Jean-Pierre Digard

2. Cf., par exemple, Robert Capot-Rey, Géographie de la circulation sur les continents, Paris, Gallimard, 1946 ("Géographie humaine» 20) : 31-48 et 159191, et la vaste synthèse de Xavier de Planhol \& Pierre Rognon, Les Zones tropicales arides et subtropicales, Paris, Armand Colin, 1970 ( U. Série Géographie»): 252-289 et 373-387.

3. Douglas L. Johnson, The Nature of Nomadism. A Comparative Study of Pastoral Migrations in Southwestern Asia and Northern Africa, Chicago, University of Chicago, 1969 ("Research Paper» 118). 4. Carole Ferret, «Discontinuités spatiales et pastoralisme nomade en Asie intérieure au tournant des $\mathrm{XIX}^{\mathrm{e}}$ et $\mathrm{XX}^{\mathrm{e}}$ siècles", Annales. Histoire, sciences sociales, 2014, 69 (4) : 957-996 (https://www.cairn.info/ revue-annales-2014-4-page-957.htm). 